\title{
Monitoring the Disassembly of Virus-like Particles by ${ }^{19} \mathrm{~F}-\mathrm{NMR}$
}

Rogen L. C. Leung, ${ }^{\dagger}$ Matthew D. M. Robinson, ${ }^{\dagger}$ Alaa A. A. Ajabali, Gogulan Karunanithy, Brian Lyons, Ritu Raj, Saeed Raoufmoghaddam, Shabaz Mohammed, ${ }^{\circledR}$ Timothy D. W. Claridge, ${ }^{\circledR}$ Andrew J. Baldwin, and Benjamin G. Davis*

Chemistry Research Laboratory, Department of Chemistry, University of Oxford, Mansfield Road, Oxford OX1 3TA, United Kingdom

\section{Supporting Information}

ABSTRACT: Virus-like particles (VLPs) are stable protein cages derived from virus coats. They have been used extensively as biomolecular platforms, e.g., nanocarriers or vaccines, but a convenient in situ technique is lacking for tracking functional status. Here, we present a simple way to monitor disassembly of ${ }^{19}$ F-labeled VLPs derived from bacteriophage $\mathrm{Q} \beta$ by ${ }^{19} \mathrm{~F}$ NMR. Analysis of resonances, under a range of conditions, allowed determination not only of the particle as fully assembled but also as disassembled, as well as detection of a degraded state upon digestion by cells. This in turn allowed mutational redesign of disassembly and testing in both bacterial and mammalian systems as a strategy for the creation of putative, targeted-VLP delivery systems.

$\mathrm{V}$ irus-like particles (VLPs) are icosahedral protein cages made up of hundreds of capsid protein subunits from different viruses. They have well-defined structures and can be strikingly stable under extremes of temperature, ${ }^{1,2} \mathrm{pH}^{2}$ and in different solvents. ${ }^{3}$ These render them potentially suitable for encapsulating materials such as proteins, ${ }^{4,5}$ synthetic polymers, ${ }^{6,7}$ oligonucleotides, ${ }^{8,9}$ and smaller molecules. ${ }^{10,11}$ Furthermore, their protein surface can be used to append different functional groups, ligands or antigens for targeting, ${ }^{12-14}$ imaging, ${ }^{15}$ vaccination, $^{16,17}$ and other biomedical ${ }^{18,19}$ purposes. Cellular internalization of VLP has been predominantly determined by fluorescence microscopy ${ }^{12,20}$ and/or transmission electron microscopy (TEM). ${ }^{21,22}$ Although these techniques provide robust data on the position of particles relative to target cells, they do not provide much information on, e.g., disassembly status. The latter can be particularly important when VLPs are applied as carriers, as cargo release is greatly dependent on breakdown. Thus, it would be useful to develop a convenient way for monitoring the multimeric state of VLPs to aid their design toward such functional goals.

${ }^{19} \mathrm{~F}$-protein labeling can prove invaluable because ${ }^{19} \mathrm{~F}$ generally has a very low background in biological samples. It is NMR-active with a wide chemical shift range making it sensitive to the local environment, and has a high sensitivity, making it easy to detect; ${ }^{23}$ both useful for monitoring structural and interaction changes. Thus, "background-free" virus tracking in vivo could use ${ }^{19}$ F-NMR without obscurity from the complex mixture of biomacromolecules in the cell. We envisaged that labeling VLPs with a ${ }^{19} \mathrm{~F}$-containing unnatural amino acid (uAA) would allow us to monitor structural change of particles at a molecular level via ${ }^{19} \mathrm{~F}-\mathrm{NMR}$.

The VLP derived from the bacteriophage $\mathrm{Q} \beta$ is formed from 180 copies of a 132 amino acid subunit ${ }^{24}$ and was chosen as a model for the introduction. $\mathrm{Q} \beta$-VLP is considered to be more stable compared with other VLPs, such as MS2, due to intersubunit disulfide linkages. ${ }^{24}$ One approach to introducing uAAs involves the commandeering of "sense" codons for amino acids such as methionine (Met) to incorporate Met analogs; ${ }^{25-28}$ trifluoroMet $(\mathrm{Tfm})$ was chosen for close structural similarity, relatively high F content (and so NMR sensitivity), and F magnetic equivalence (and so simpler, stronger signal). $\mathrm{Tfm}$ has been used to probe enzyme mechanism ${ }^{27,29}$ and suggested as a residue that allows creation of unusual physicochemical properties. ${ }^{30}$ Wild-type (WT) $\mathrm{Q} \beta$ contains no Met sites, thus conversion of the Lys 16 codon in the $\mathrm{Q} \beta$ gene to Met codon would allow site-specific incorporation. Site 16 is one of the most exposed on the particle, ${ }^{31}$ and we reasoned would also provide an excellent probe site (Figure 1a).

Expression of this gene in Escherichia coli Met-auxotroph B834(DE3) in the presence of Tfm $(\sim 1.7 \mathrm{mM})$ under optimized conditions (see SI) allowed the production of $\mathrm{Q} \beta$-Lys16Tfm ("Q $\beta$-F") with $\sim 85 \%$ F-incorporation (Figure $1 \mathrm{~b}$ ), a level consistent with prior levels in other proteins; ${ }^{29}$ Met competes well with Tfm for the methionyl-tRNA synthetase (MetRS) ${ }^{32}$ and even after exhaustive Met depletion $15 \%$ is incorporated. The integrity of VLPs formed from the self-assembly of expressed $\mathrm{Q} \beta$ F was confirmed by both dynamic light scattering (DLS) (Figure 1c) and TEM (Figure 1d). Their measured radius (15.3 \pm 0.6 $\mathrm{nm}$ ) was found to be identical to WT within experimental error (Figure S2).

When these intact $\mathrm{Q} \beta$-F VLPs were analyzed by ${ }^{19} \mathrm{~F}-\mathrm{NMR}$, a broad resonance (full width at half height, $\mathrm{FWHH}=\sim 240 \mathrm{~Hz}$ ) was observed with a shift of $-40.68 \mathrm{ppm}$, and a remarkably large $\mathrm{R}_{2}$ value of $760 \mathrm{~s}^{-1}$ (Figure 2a and Figure S16). Controlled disassembly of the particles was achieved through titrated addition of denaturant and reductant ${ }^{33}$ and monitored by ${ }^{19} \mathrm{~F}$ NMR. Denaturant SDS $(0.2 \mathrm{M})$ gave a single sharp peak (FWHH $=12.6 \mathrm{~Hz}$ ) with a shift of $-40.90 \mathrm{ppm}$, and an $\mathrm{R}_{2}$ value of $8 \mathrm{~s}^{-1}$ (Figure S16); the significant change of chemical shift and peak width suggested clear change of particle structure. Reductant DTT alone had negligible effect. This was confirmed by nativePAGE (dissemination of corresponding bands); when further

Received: October 27, 2016

Published: March 28, 2017 
(a)

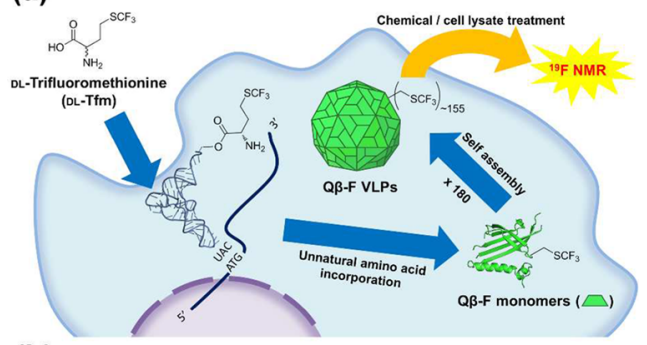

(b)

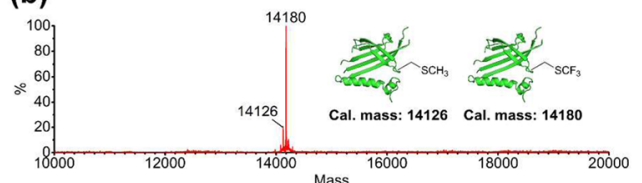

(c)

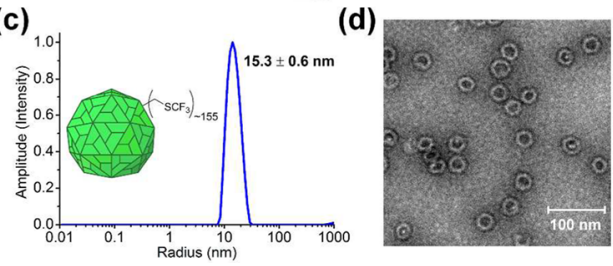

Figure 1. (a) Cellular strategy for genetic incorporation of $\mathrm{Tfm}$ into $\mathrm{Q} \beta$. (b) Reducing ES-MS of $\mathrm{Q} \beta$-F (raw ion series, Figure $\mathrm{S} 1 \mathrm{a}, \mathrm{b}$ ) shows $\sim 85 \%$ $\mathrm{Tfm}$ incorporation. Both DLS (c) and TEM (d) reveal fully assembled discrete particles.
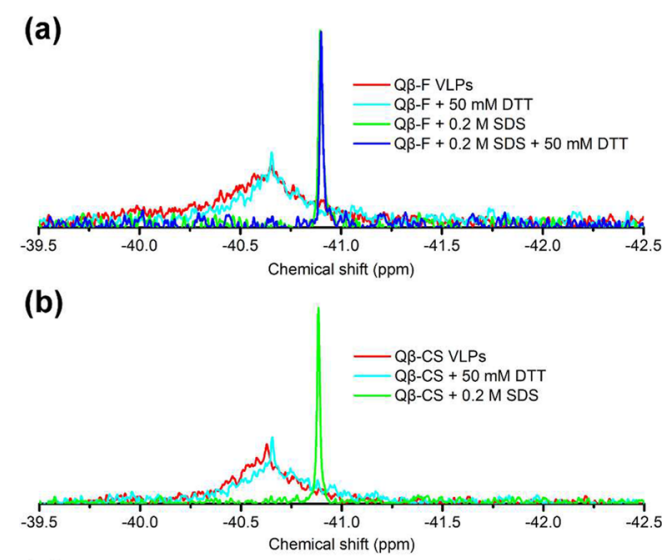

(c)

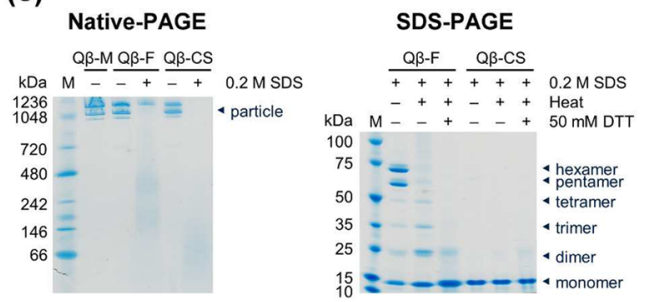

Figure 2. ${ }^{19} \mathrm{~F}-\mathrm{NMR}$ spectra of (a) $\mathrm{Q} \beta$-F VLPs; (b) $\mathrm{Q} \beta$-CS VLPs under different conditions. 1,1,1-Trifluoroacetone (TFA) was added to all of the samples as an internal reference. The reference peak was fixed to $-86.40 \mathrm{ppm}$ relative to $\mathrm{CFCl}_{3}$. Intensity values have been rescaled for comparison (see Figure S3). (c) Left: Native-PAGE. Under nondenaturing conditions, the particles have low mobility, consistent with a fully assembled form. In the presence of $0.2 \mathrm{MSDS}$, the virus particles are destabilized. Right: SDS-PAGE. Under denaturing conditions, a range of oligomeric states are observed, stabilized by persistent disulfide bonds. $\mathrm{M}$, protein marker; $\mathrm{Q} \beta$-M, $\mathrm{Q} \beta$-K16 M intact VLPs; heat, $90{ }^{\circ} \mathrm{C}, 60 \mathrm{~min}$. Gel stain: Coomassie Blue. analyzed under denaturing conditions by SDS-PAGE, hexamers and pentamers were observed (Figure 2c), consistent with persistent disulfide bonds in subassemblies. Further treating the same sample with reductant dithiothreitol (DTT) led to formation of monomers (Figure $2 \mathrm{c}$, right), characterized by similar chemical shift and peak width to the 5/6-mers $\left(\delta_{\mathrm{F}}=\right.$ -40.90 ppm; FWHH $=\sim 7 \mathrm{~Hz}$, Figure 2a). Taken together, these results reveal that we can monitor the assembly state of the VLPs using ${ }^{19} \mathrm{~F}$ NMR.

Next, we exploited this ability to determine particle-state as a guide to particle design. As a proof of concept, we set out to develop a more "flimsy" VLP system that might allow more ready or rapid cargo release. Our ${ }^{19} \mathrm{~F}-\mathrm{NMR}$ studies on $\mathrm{Q} \beta$-F had suggested a role for reducible cross-linking. We therefore generated a triple mutant $(\mathrm{Q} \beta-\mathrm{K} 16 \mathrm{M}-\mathrm{C} 74 \mathrm{~S}-\mathrm{C} 80 \mathrm{~S})$ in which the Cys 74 and Cys80 that contribute to intersubunit disfulfide covalent cross-linking were converted to isosteric but nonreactive Ser. Tfm was incorporated into protein as for $\mathrm{Q} \beta$-F to generate named $\mathrm{Q} \beta$-K16Tfm-C74S-C80S (named $\mathrm{Q} \beta$-CS, Figure S4). Pleasingly, $\mathrm{Q} \beta$-CS still formed discrete particles, even without disulfide bonds at C74 and C80 (Figure S2), consistent with prior studies that have identified the dominant contribution of noncovalent interactions between viral subunits. ${ }^{34}$ The relative robustness of these $\mathrm{Q} \beta$-CS VLPs was probed with ${ }^{19} \mathrm{~F}$-NMR: in contrast to $\mathrm{Q} \beta$-F, $\mathrm{Q} \beta$-CS VLPs disassembled directly into monomers under denaturing conditions when treated only with SDS (Figure 2c), consistent with our intended design. The chemical shifts of $\mathrm{Q} \beta$-CS VLPs in the absence and presence of SDS were highly similar $(-40.63 \mathrm{ppm}, \mathrm{FWHH}=\sim 200 \mathrm{~Hz}$; $-40.88 \mathrm{ppm}, \mathrm{FWHH}=\sim 6 \mathrm{~Hz}$, respectively) to those observed for $\mathrm{Q} \beta$ (Figure $2 \mathrm{~b}$ ). Reductant DTT alone had negligible effect.

We further characterized multimer and monomers using ${ }^{19} \mathrm{~F}$ diffusion NMR (Figure 3 and Figure S5). ${ }^{35}$ Despite their similar

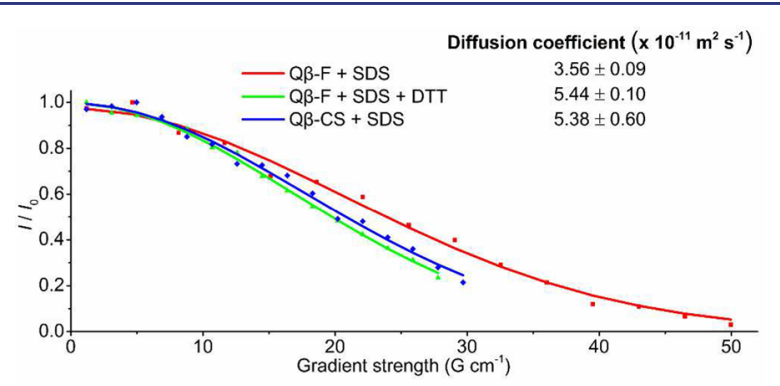

Figure 3. $I / I_{0}$ versus gradient strength of various $\mathrm{Q} \beta$ species. Diffusion coefficients were calculated using TopSpin and normalized according to TFA reference to eliminate environmental or solvent effects (Table S1).

chemical shifts in $1 \mathrm{D}{ }^{19}$ F-NMR spectra, their diffusion coefficients were different. Notably, adding DTT in addition to SDS to the solution resulted in an increase in diffusion coefficient, consistent with destabilizing subassemblies, and the value from $[\mathrm{Q} \beta-\mathrm{F}]+\mathrm{SDS}+\mathrm{DTT}$ was essentially identical to that from $[\mathrm{Q} \beta$ CS $]+$ SDS, as expected, as well as $\mathrm{Q} \beta$-F monomer prevented from disulfide formation by Cys-alkylation (Table S1). Diffusion coefficients for the full particle were challenging to measure owing to the very large $\mathrm{R}_{2}$ of the resonance (Figure $2 \mathrm{a}$ and Figure S5). The values obtained were significantly smaller than measured for degraded assemblies (in the presence of SDS and DTT) but larger than expected for full size particle, suggesting that they stem from high order multimers only observed in the presence of intact particles, thereby allowing us to monitor assembly state of the VLPs. 
Importantly, this validation of particle disassembly states also allowed us to determine the extent of particle dissociation and to test putative disassembly pathways. Thus, gradual titration of $\mathrm{Q} \beta$ $F$ with SDS led to a gradual diminution in the intensity of the broad particle peak $\left(\delta_{\mathrm{F}}-40.7 \mathrm{ppm}\right)$ with simultaneous emergence in intensity of the peak ascribed to disassembled species (Figure S6). Interestingly, this suggests that the mechanism of disassembly in the presence of denaturant is not a cooperative catastrophic collapse but a gradual drift in population in response to environmental stress.

Next, we tested this ability to monitor particle state in a biological context (Figure $4 \mathrm{a}$ ). $\mathrm{Q} \beta$-F VLPs were introduced to a

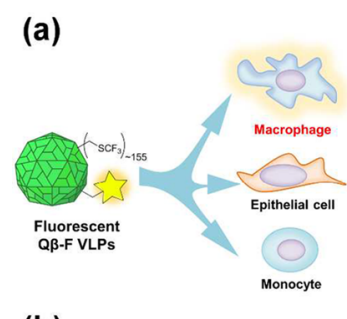

(b)
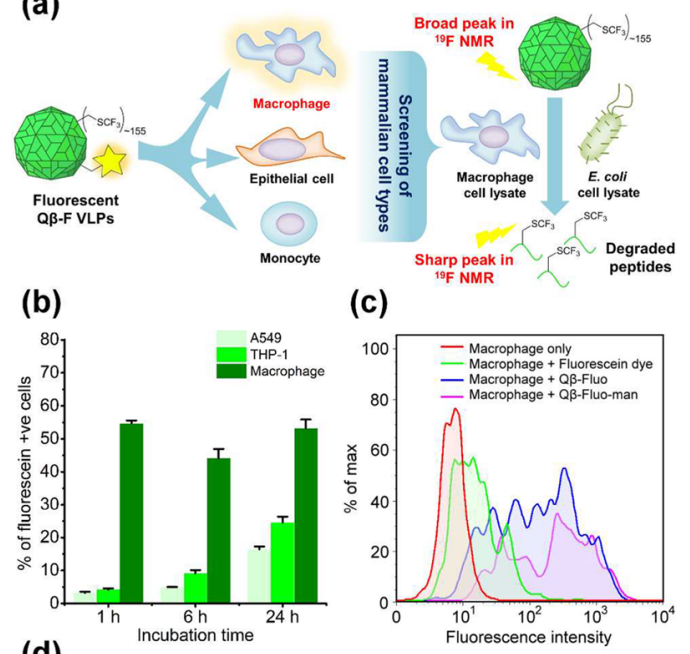

(c)

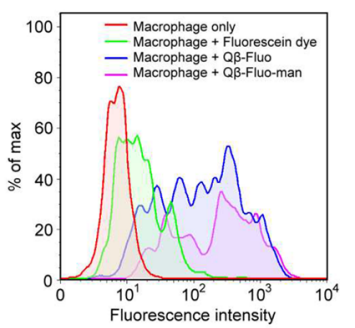

(d)

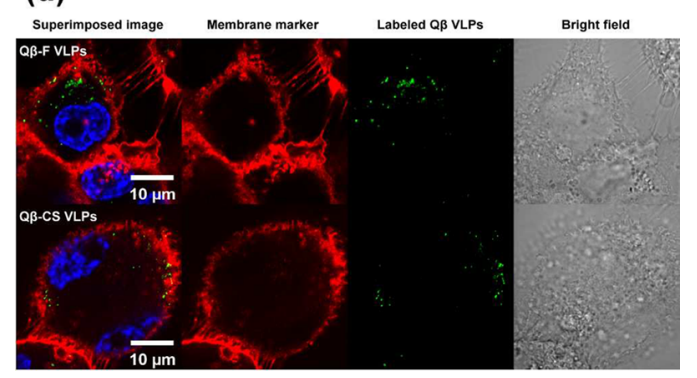

Figure 4. (a) Multimodal tracking of VLP response to cells. VLPs were screened for both internalization and stability to corresponding internal cellular fluid (lysate) using combined fluorescent and ${ }^{19} \mathrm{~F}$-labeling. (b) Flow cytometry determined efficiency of mammalian cell internalization of $\mathrm{Q} \beta$-F VLPs. (c) FACS histograms for internalization of $\mathrm{Q} \beta$-F and mannose-decorated $\mathrm{Q} \beta$-F VLPs into macrophage cells after $24 \mathrm{~h}$ incubation. (d) Microscopy of macrophage with fluorescein-labeled $\mathrm{Q} \beta$ $\mathrm{F}$ or $\mathrm{Q} \beta$-CS VLPs after $20 \mathrm{~min}$ of incubation.

range of representative mammalian cell types: A549 epithelial cells, THP-1 monocytes, and THP-1-derived macrophages. Flow cytometry (Figure S9) and confocal microscopy (Figure S10), following chemical, fluorescent, surface-labeling (Figures S7S9), revealed that only THP-1-derived macrophages readily engulfed VLPs (Figure 4b,c). Confocal fluorescence microscopy confirmed internalization (Figure $4 \mathrm{~d}$ and Figure S11); observations after $20 \mathrm{~min}$ suggested entry via an endosomal pathway (Figure S12).

To assess the more detailed behavior of VLPs in the milieu of the cell interior, we incubated VLPs with cell lysates and probed their behavior by ${ }^{19} \mathrm{~F}-\mathrm{NMR}$. In addition to the internalization by THP-1-derived macrophages discovered here, E. coli is the natural host cell of $\mathrm{Q} \beta$ virus; lysates from both "host” cell types were therefore chosen (Figure $4 \mathrm{a}$ ). In the viscous cellular environment, it is expected that all ${ }^{19} \mathrm{~F}-\mathrm{NMR}$ resonances would be broadened due to macromolecular crowding. ${ }^{36}$ Nonetheless, we were still able to observe the broad resonance thereby allowing monitoring of intact virus assemblies in both lysates, owing to the particular suitability of the pairing of ${ }^{19} \mathrm{~F}$ NMR and our system (Figure 5a and S13). Upon prolonged incubation at
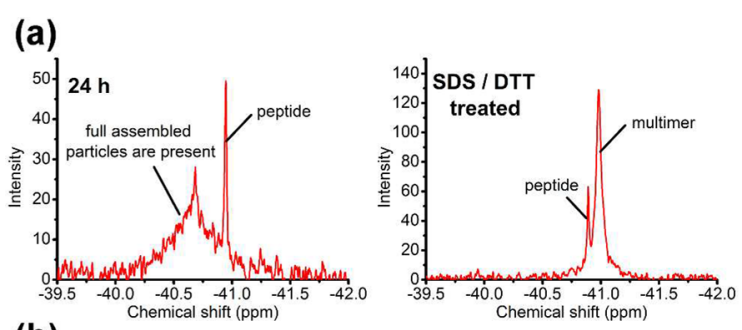

(b)
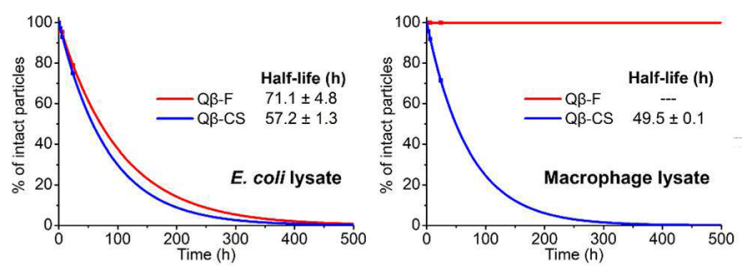

Figure 5. (a) ${ }^{19} \mathrm{~F}-\mathrm{NMR}$ of $\mathrm{Q} \beta$-F incubated with E. coli lysate at $37{ }^{\circ} \mathrm{C}$ for $24 \mathrm{~h}$ (left), followed by SDS+DTT treatment (right). (b) Decay curves of $\mathrm{Q} \beta$-F and $\mathrm{Q} \beta$-CS VLPs in E. coli or macrophage cell lysates.

$37^{\circ} \mathrm{C}$, a sharp peak with a shift of $-40.95 \mathrm{ppm}(\mathrm{FWHH}=9.9 \mathrm{~Hz})$ appeared, with increasing intensity over time. NMR measurements determined the diffusion coefficient to be $3.0 \times 10^{-10} \mathrm{~m}^{2}$ $\mathrm{s}^{-1}$ (Table S2), suggesting an even smaller species than those found previously. This was confirmed upon additional chemical denaturation (added SDS+DTT): the resonance from this smaller species was readily discernible from the disassembled species observed previously (Figure 5a and Figure S13). Together, these data suggest: (i) the formation of smaller (monomeric or peptidic) Tfm-containing fragments of $\mathrm{Q} \beta$ derived from lysate (likely mediated by proteolysis) and (ii), importantly, that our developed system can distinguish between intact particles, intervening multimeric states and a further stage of disassembly (or degradation) generated within complex milieu such as cell lysates.

In turn, this system, vitally, allowed us to analyze our designed "flimsy" VLP system $\mathrm{Q} \beta$-CS. Comparison of the degradation processes of $\mathrm{Q} \beta$-F and $\mathrm{Q} \beta$-CS revealed a significantly shorter half-life for $\mathrm{Q} \beta$-CS in both lysate systems (Figure $5 \mathrm{~b}$ ), consistent with in vitro data and with the designed removal of disulfide bonds mediated by Cys 74 and Cys 80 . Interestingly, whereas both $\mathrm{Q} \beta$-F and $\mathrm{Q} \beta$-CS were disassembled by E. coli, only the more stable $\mathrm{Q} \beta$-F was not significantly disassembled by the macrophage cells. Consistent with these observations, VLP-derived peptides were directly identified by MS/MS; time course analyses revealed formation (including $\mathrm{Tfm}$-containing peptides) and further digestion into fragments. Notably, more peptides were observed from "weakened" $\mathrm{Q} \beta$-CS in both E. coli and macrophage (Figures S17-S19).

Finally, to illustrate the potential of these VLP platforms for further elaboration, we tuned their cellular uptake. Chemicalmodification with putative ligands for cell-surface receptors as "cell-targeting" groups allowed a proof-of-principle of such 
further adaptation. Thus, we extended the tropism of $\mathrm{Q} \beta$-F VLPs from $E$. coli and macrophages toward other cells through surface chemical-attachment ${ }^{37}$ of D-mannosyl residues; these alterations increased uptake of VLPs by THP-1 monocytes (Figures S14 and S15).

In summary, we have established a system for monitoring the disassembly of VLPs using ${ }^{19}$ F-NMR (see Figure S16 for further discussion) that is applicable even in complex biological milieu. Successful application to design, tested here in such "real" systems, suggests its potential application in yet more complex, e.g., in vivo systems. We have also shown here the potential for dual (fluorophore and ${ }^{19} \mathrm{~F}$ )-labeling and corresponding dualmode interrogation as well as combination with chemicalmodification "cell-targeting" methods. We postulate that the strategy of designed, mechanistically verified alteration of $\mathrm{Q} \beta$ VLPs shown here might serve as an example methodological workflow for functional design of other future, designed viral, VLP and/or biological nanoparticle systems. Notably, it has been suggested ${ }^{38}$ that antibacterial phage strategies may benefit from novel mutant phages, including designed variants such as those that we have explored here. We have shown that a form of cellselectivity (E. coli vs macrophage) may be engineered here, allowing possible application to target, e.g., intramacrophage bacteria; future experiments will explore this and related potential.

\section{ASSOCIATED CONTENT}

\section{S Supporting Information}

The Supporting Information is available free of charge on the ACS Publications website at DOI: 10.1021/jacs.6b11040.

Experimental details and spectroscopic data (PDF) Tables of QB peptides (XLSX)

\section{AUTHOR INFORMATION}

\section{Corresponding Author}

*Ben.Davis@chem.ox.ac.uk

\section{ORCID}

Shabaz Mohammed: 0000-0003-2640-9560

Timothy D. W. Claridge: 0000-0001-5583-6460

Benjamin G. Davis: 0000-0002-5056-407X

\section{Author Contributions}

${ }^{\dagger}$ R.L.C.L. and M.D.M.R. contributed equally.

\section{Notes}

The authors declare no competing financial interest.

\section{ACKNOWLEDGMENTS}

We thank CRUK, EPSRC (M.D.M.R.) BBSRC (A.J.B. David Phillip's fellowship BB/J014346/1), BHF (AAAA) for funding; Prof C. J. Schofield for advice and Prof M. G. Finn for $\mathrm{Q} \beta$-WT plasmid.

\section{REFERENCES}

(1) Ashcroft, A. E.; Lago, H.; Macedo, J. M.; Horn, W. T.; Stonehouse, N. J.; Stockley, P. G. J. Nanosci. Nanotechnol. 2005, 5, 2034.

(2) Ausar, S. F.; Foubert, T. R.; Hudson, M. H.; Vedvick, T. S.; Middaugh, C. R. J. Biol. Chem. 2006, 281, 19478.

(3) Johnson, H. R.; Hooker, J. M.; Francis, M. B.; Clark, D. S. Biotechnol. Bioeng. 2007, 97, 224.

(4) Wu, Y.; Yang, H.; Shin, H.-J. MRS Online Proc. Libr. 2011, 1317, rr03.

(5) Lee, K. W.; Tan, W. S. J. Virol. Methods 2008, 151, 172.
(6) Hu, Y.; Zandi, R.; Anavitarte, A.; Knobler, C. M.; Gelbart, W. M. Biophys. J. 2008, 94, 1428.

(7) Minten, I. J.; Ma, Y.; Hempenius, M. A.; Vancso, G. J.; Nolte, R. J. M.; Cornelissen, J. J. L. M. Org. Biomol. Chem. 2009, 7, 4685.

(8) Cooper, A.; Shaul, Y. Biochem. Biophys. Res. Commun. 2005, 327, 1094.

(9) Galaway, F. A.; Stockley, P. G. Mol. Pharmaceutics 2013, 10, 59.

(10) Brasch, M.; de la Escosura, A.; Ma, Y.; Uetrecht, C.; Heck, A. J. R.; Torres, T.; Cornelissen, J. J. L. M. J. Am. Chem. Soc. 2011, 133, 6878.

(11) Wu, W.; Hsiao, S. C.; Carrico, Z. M.; Francis, M. B. Angew. Chem., Int. Ed. 2009, 48, 9493.

(12) Banerjee, D.; Liu, A. P.; Voss, N. R.; Schmid, S. L.; Finn, M. G. ChemBioChem 2010, 11, 1273.

(13) Lee, K. W.; Tey, B. T.; Ho, K. L.; Tan, W. S. J. Appl. Microbiol. 2012, 112, 119.

(14) Pan, Y.; Zhang, Y.; Jia, T.; Zhang, K.; Li, J.; Wang, L. FEBS J. 2012, 279, 1198.

(15) Stephanopoulos, N.; Carrico, Z. M.; Francis, M. B. Angew. Chem., Int. Ed. 2009, 48, 9498.

(16) Yin, Z.; Chowdhury, S.; McKay, C.; Baniel, C.; Wright, W. S.; Bentley, P.; Kaczanowska, K.; Gildersleeve, J. C.; Finn, M. G.; BenMohamed, L.; Huang, X. ACS Chem. Biol. 2015, 10, 2364.

(17) Low, J. G. H.; Lee, L. S.; Ooi, E. E.; Ethirajulu, K.; Yeo, P.; Matter, A.; Connolly, J. E.; Skibinski, D. A. G.; Saudan, P.; Bachmann, M.; Hanson, B. J.; Lu, Q.; Maurer-Stroh, S.; Lim, S.; Novotny-Diermayr, V. Vaccine 2014, 32, 5041.

(18) Steinmetz, N. F.; Hong, V.; Spoerke, E. D.; Lu, P.; Breitenkamp, K.; Finn, M. G.; Manchester, M. J. Am. Chem. Soc. 2009, 131, 17093.

(19) Ribeiro-Viana, R.; Sanchez-Navarro, M.; Luczkowiak, J.; Koeppe,

J. R.; Delgado, R.; Rojo, J.; Davis, B. G. Nat. Commun. 2012, 3, 1303.

(20) Welsher, K.; Yang, H. Nat. Nanotechnol. 2014, 9, 198.

(21) Lewis, J. D.; Destito, G.; Zijlstra, A.; Gonzalez, M. J.; Quigley, J. P.; Manchester, M.; Stuhlmann, H. Nat. Med. 2006, 12, 354.

(22) Ewers, H.; Smith, A. E.; Sbalzarini, I. F.; Lilie, H.; Koumoutsakos, P.; Helenius, A. Proc. Natl. Acad. Sci. U. S. A. 2005, 102, 15110.

(23) Kitevski-LeBlanc, J. L.; Prosser, R. S. Prog. Nucl. Magn. Reson. Spectrosc. 2012, 62, 1.

(24) Golmohammadi, R.; Fridborg, K.; Bundule, M.; Valegard, K.; Liljas, L. Structure 1996, 4, 543.

(25) van Hest, J. C. M.; Kiick, K. L.; Tirrell, D. A. J. Am. Chem. Soc. 2000, $122,1282$.

(26) Kiick, K. L.; Saxon, E.; Tirrell, D. A.; Bertozzi, C. R. Proc. Natl. Acad. Sci. U. S. A. 2002, 99, 19.

(27) Duewel, H.; Daub, E.; Robinson, V.; Honek, J. F. Biochemistry 1997, 36, 3404.

(28) Bogosian, G.; Violand, B. N.; Dorwardking, E. J.; Workman, W. E.; Jung, P. E.; Kane, J. F. J. Biol. Chem. 1989, 264, 531.

(29) Holzberger, B.; Rubini, M.; Möller, H. M.; Marx, A. Angew. Chem., Int. Ed. 2010, 49, 1324.

(30) Neil, E.; Marsh, G. Chem. Biol. 2000, 7, R153.

(31) Shepherd, C. M.; Borelli, I. A.; Lander, G.; Natarajan, P.; Siddavanahalli, V.; Bajaj, C.; Johnson, J. E.; Brooks, C. L., 3rd; Reddy, V. S. Nucleic Acids Res. 2006, 34, D386.

(32) Crepin, T.; Schmitt, E.; Mechulam, Y.; Sampson, P. B.; Vaughan, M. D.; Honek, J. F.; Blanquet, S. J. Mol. Biol. 2003, 332, 59.

(33) Takamatsu, H.; Iso, K. Nature 1982, 298, 819.

(34) Fiedler, J. D.; Higginson, C.; Hovlid, M. L.; Kislukhin, A. A.; Castillejos, A.; Manzenrieder, F.; Campbell, M. G.; Voss, N. R.; Potter, C. S.; Carragher, B.; Finn, M. G. Biomacromolecules 2012, 13, 2339.

(35) Morris, G. A. In eMagRes.; John Wiley \& Sons, Ltd, 2007.

(36) Ellis, R. J. Curr. Opin. Struct. Biol. 2001, 11, 114.

(37) Pearce, O. M.; Fisher, K. D.; Humphries, J.; Seymour, L. W.; Smith, A.; Davis, B. G. Angew. Chem., Int. Ed. 2005, 44, 1057.

(38) Matsuzaki, S.; Uchiyama, J.; Takemura-Uchiyama, I.; Daibata, M. Nature 2014, 509, S9. 